\title{
Diagnostic Efficacy of Pelvic Ultrasound in Central Precocious Puberty in Girls: a Retrospective Cohort Study
}

Elham Zarei

Iran University of Medical Sciences

Nima Rakhshankhah ( $\nabla$ nima3rh@gmail.com )

Iran University of Medical Sciences https://orcid.org/0000-0001-6708-0904

Mahmoud Khodadost

Iran University of Medical Sciences

Abolfazl Abouie

Iran University of Medical Sciences

Kosar Mohammadnejad

Tehran University of Medical Sciences

Mehdi Vafadar

Iran University of Medical Sciences

\section{Research}

Keywords: Central precocious puberty, isolated premature thelarche, isolated premature adrenarche, pelvic ultrasound, diagnosis

Posted Date: June 1st, 2021

DOl: https://doi.org/10.21203/rs.3.rs-552824/v1

License: (c) (i) This work is licensed under a Creative Commons Attribution 4.0 International License. Read Full License 


\section{Abstract}

Background: Differentiating central precocious puberty (CPP) patients from normal cases and CPP-like patients "isolated premature thelarche (IPA) and isolated premature thelarche (IPT)" is important for beginning of treatment. Although the GnRH stimulation test is considered the gold standard for diagnosis of CPP, Because of its wide limitations, our study targets to evaluate pelvic sonography parameters as a contributory tool for CPP diagnosis.

Methods: We consecutively enrolled 183 cases (93 CPP, 16 IPT, 12 IPA and 62 of age-matched normal controls) in our study over four years. All cases are classified by clinical and laboratory findings and are followed up for at least 2 years. Pelvic sonography parameters included uterine fundus, body and cervix anteroposterior diameter, fundus/cervix ratio, uterine length and transverse diameter, uterine volume, endometrial thickness, ovarian volumes and diameter of the largest follicle are evaluated in all classified groups. One-way ANOVA, post hoc and receiver operating characteristic (ROC) analysis was used to compare the study groups.

Results: Our study found that all sonography parameters differ significantly between CPP and normal control cases, also a significant difference is found between CPP compared to IPT or IPA cases in all parameters except in cervix anteroposterior diameter, ovarian volumes and diameter of the largest follicle. In order of best parameters for differentiating CPP compared to study groups, uterine volume (a cut-off of $1.40 \mathrm{ml}$ had a sensitivity of $75.27 \%$ and a specificity of $75.56 \%$ ), transverse diameter (a cut-off of 13.5 $\mathrm{mm}$ had a sensitivity of $72.04 \%$ and a specificity of $71.11 \%$ ) and $\mathrm{F} / \mathrm{C}$ ratio (a cut-off of 0.98 had a sensitivity of $78.49 \%$ and a specificity of $70 \%$ ) was selected. Our study also classified sonography parameters as in equal diagnostic value to uterine volume (as the best diagnostic parameter with area under the curve of 0.826 ) and not equal diagnostic value to uterine volume.

Conclusions: Pelvic Sonography parameters may improve the diagnosis of CPP patients and can have a contributory role in distinguishing treatment needed patients from other patients. The best diagnostic parameter and its cut-off value could change according to different ethnicities and studies.

\section{Background}

Girl's precocious puberty is defined as development of secondary sexual characteristics, growth spurts, and psychosocial changes before the age of 8 years in white or Caucasian people and before the age of 7 years in black or African American people (1-3). Precocious puberty is classified into two main groups which is defined as gonadotropin dependent precocious puberty (GDPP) or gonadotropin independent precocious puberty (GIPP). Conditions such as gonadal, adrenal endocrine disorders, exogenous hormone consumption, pseudo precocious-puberty and peripheral precocious puberty is classified as GIPP group. On the other hand GDPP group which is called central precocious puberty (CPP) is caused by hypothalamic-pituitary-gonadal (HPG) axis activity and includes $58-90 \%$ of the precocious puberty cases(4). CPP is mostly caused by idiopathic reasons and in rare cases brain abnormalities such as 
tumors, trauma, infections and malformations are the main causes of HPG axis activity(5). Although the CPP cases occur at a younger age, but the pattern of natural maturation stages, such as thelarche, pubarche and menarche, occurs the same as normal people. The main concerns about CPP are premature bone maturation, reduced final patient height, and physiological stress which is imposed on the patients $(6,7)$. That's why timely initiation of CPP treatment with $\mathrm{GnRH}$ (gonadotropin releasing hormone) agonists is of great value for patients (8). Distinguishing CPP cases from isolated premature thelarche (IPT) and isolated premature adrenarche (IPA) is clinically important because despite the onset of sexual characteristics similar to CPP, they don't accelerate bone maturation and does not require treatment with $\mathrm{GnRH}$ agonists(9). Furthermore differentiating CPP cases from pseudo-precocious puberty and exogenous obesity, which its prevalence has been increasing in recent years, is important. it is possible that in cases of obesity, bone age may be higher than normal, but there is no other evidence of puberty and therefore does not require treatment with $\mathrm{GnRH}$ agonists(10). Clinical diagnosis of CPP is based on physical examination, bone age, and growth rate(11). GnRH stimulation test as the gold standard is used to differentiate of CPP patients from other cases which are considered suspicious and borderline, but despite its high specificity, it has disadvantages such as low sensitivity, time consuming and requires multiple blood sampling(12). Pelvic ultrasonography (US) is almost always requested in cases of clinical suspicion of any type of precocious puberty. Pelvic US has an advantage of being noninvasive, accessible, radiation-free and inexpensive, and it is a useful diagnostic tool for assessing female's pelvic(13). Pelvic US gives detailed information about uterine and ovarian size, fundus to cervical ratio, endometrial thickness, follicle size and distribution of the ovaries. Pelvic US can also help to diagnose cysts and pelvic masses. Numerous studies have been performed on increasing the volume of the ovaries and uterus and increasing the size and number of follicles during the years leading up to puberty. Also numerous attempts have been made to use US to distinguish normal girls from girls with precocious puberty. But still diagnostic worth of pelvic US parameters remain unclear. Some studies report overlapping values of pelvic US parameters between CPP and other groups(14) and in other studies pelvic US parameters are useful for differentiating CPP from normal individuals, nevertheless different studies determine different cut-off points for sonography parameters $(15,16)$.

This study aimed to evaluate the usefulness of pelvic ultrasonography and its parameters in differentiating girls with CPP from normal individuals with or without suspicious characteristics. Therefore, in this study an attempt is made to compare the parameters of the pelvic ultrasonography between the CPP, IPT, IPA and normal individuals to determine safe cut-off limits for each of the parameters.

\section{Methods}

Study population

The cases of this study were female patients with eight or less than eight years old who were referred to the Endocrinology department of our hospital (Tehran Ali Asghar children's hospital) for evaluation of precocious puberty from 2015 to 2019 years. Four groups are labeled as control, IPT, IPA, and CPP groups 
with exclusion criteria of CNS abnormalities related endocrinological abnormalities such as adrenal, gonadal, and thyroidal abnormalities, iatrogenic or exogenous hormonal contact, and also patients with no proper follow-up. The study was confirmed by the local ethics committee and written informed parental consent, following the revised Helsinki declaration, was given to all participants. All of the cases mentioned below are clinically and axiologically followed and confirmed for at least two years.

- Control group

Sixty-two girls $\leq 8$ years old are included in this group after the exclusion of 3 of them due to noted exclusion criteria (no proper follow-up). The inclusion criteria for the control group were as follows: (1) 8 or less than 8 years for chronological age, (2) children without secondary sexual characteristics, (3) children within 2 SD of bone age and normal height velocity, (4) children who have hormonal assessments and pelvic ultrasonography in our hospital.

- CPP (central precocious puberty) group

Ninety-three $\leq 8$ years old are included in this group after the exclusion of 4 of them due to noted exclusion criteria (two of them have hypophyseal microadenoma, one has hypothyroidism and one uses estrogen cream treatment for labial adhesion in infancy). The inclusion criteria for the CPP group were as follows: (1) 8 or less than 8 years for chronological age, (2) children with secondary sexual characteristics, (3) children with more than 2 SD of bone-chronological age and accelerated height velocity, (4) children who have confirmed precocious puberty with GnRH stimulation test, (5) children who have pelvic ultrasonography in our hospital.

- IPT (isolated premature thelarche) group

Sixteen girls $\leq 8$ years old are included in this group after the exclusion of 1 of them due to adipose tissue of breast instead of real thelarche. The inclusion criteria for the IPT group were as follows (1) 8 or less than 8 years for chronological age, (2) children with breast development in the absence of any other signs of puberty, (3) children within 2 SD of bone age and normal height velocity, (4) children who have hormonal assessments and pelvic ultrasonography in our hospital.

- IPA (isolated premature adrenarche) group

Twelve girls $\leq 8$ years old are included in this group after the exclusion of 2 of them due to CAH (congenital adrenal hyperplasia) diagnosis. The inclusion criteria for the IPT group were as follows: (1) 8 or less than 8 years for chronological age, (2) children with pubic and/or axillary hair appearance in the absence of any other signs of puberty, (3) children within 2 SD of bone age and normal height velocity, (4) children who have hormonal assessments and pelvic ultrasonography in our hospital.

Study design 
All patients' secondary sexual characteristics were examined precisely and were assessed according to Tanner staging (3). Height and weight measurements and also height velocity (height gained within a year) are measured on a minimum of a six-month interval and interpreted as whether accelerated or not (17). Bone age was evaluated from the non-dominant hand radiographs and defined by Greulich scoring (18). The hormonal evaluation included basal levels of luteinizing hormone (LH), follicle-stimulating hormone (FSH), and estradiol (E2) as well as GnRH-stimulated LH and FSH levels are measured using radioimmunoassay. Stimulated LH and FSH levels are measured after 30 and $60 \mathrm{~min}$ of GnRH agent injection. Stimulated LH level of $\geq 5 \mathrm{IU} / \mathrm{L}$ was considered diagnostic for CPP in patients with pubertal characteristics (19).

Pelvic ultrasonography was done by the Radiology Department of Tehran Ali Asghar children's hospital. Philips EPIQ 7 Ultrasound Machine equipped with a 7.5-MHz linear array transducer and a 5-MHz convex array transducer was used for evaluation of patients. All patients were examined with a full bladder in a completely calm condition. Ultrasonography parameters included uterine fundus AP(anteroposterior) diameter $(\mathrm{mm})$, uterine body AP diameter $(\mathrm{mm})$, uterine cervix AP diameter $(\mathrm{mm}), \mathrm{F} / \mathrm{C}$ ratio(uterine fundus AP diameter divided by the cervix AP diameter), uterine length $(\mathrm{mm})$, uterine transverse diameter $(\mathrm{mm})$, uterine volume - calculated by the ellipse formula: $\mathrm{V}(\mathrm{ml})=$ uterine length $(\mathrm{mm}) \times$ transverse diameter $(\mathrm{mm}) \times$ AP diameter $(\mathrm{mm}) \times 0.5236$, endometrial thickness $(\mathrm{mm})$, left ovarian volume $(\mathrm{ml})$, right ovarian volume $(\mathrm{ml})$, average maximal diameter of largest follicle in both ovaries $(\mathrm{mm})$. The volume of each ovary was calculated by using the same ellipse formula as for the uterus.

Statistics

The categorical variables were presented as frequency and percent and continuous data were reported as mean and standard deviation (SD). The one-way analysis of variance (One-way ANOVA) was used to compare the mean of continuous variables between the study groups including CPP, IPT, IPA, and control group. Moreover, the post hoc analysis using the Tukey test was used for pairwise comparisons between the study groups if the initial ANOVA test was statistically significant. The Receiver-operating characteristics (ROC) analysis was used to determine the best cut-off point for ultrasonography parameters and its corresponding sensitivity, specificity, Positive Likelihood Ratio (LR+), and Negative Likelihood Ratio (LR-) for identifying patients with central precocious puberty (CPP). Furthermore, the equality of ROC area for ultrasonography parameters against the uterine volume ROC curve as a gold standard was assessed using the "Rocgold" command in Stata software. The Stata software version 12 (Stata Corp, College Station, TX) was used for statistical analysis, and a p-value less than 0.05 was considered as significant findings.

\section{Results}

Among a total of 183 children, 62 were classified as the control group (33.87\%), 93 as CPP group (50.81\%), 16 as IPT group (8.74\%), and 12 as IPA group (6.55\%).

- Comparison of the demographic characteristics and hormonal laboratory values between groups 
One-way analysis of variance and post hoc analysis used for comparison of demographic characteristics, and although there is no significant difference in chronological age and BMI of classified groups, girls with different groups had a significant difference in bone age $(p<0.001)$ and height $(p=0.003)$, in a way which, CPP group showed a significantly higher bone age (107.76 \pm 19.81 month) and taller height $(129.53 \pm 8.97 \mathrm{~cm})$ against other groups (Table 1). In one-way analysis of variance, the hormonal laboratory values showed significant differences in baseline LH $(p<0.001)$, baseline FSH $(p=0.024)$, and estradiol $(p<0.001)$ levels between classified groups, but in pairwise post-hoc analysis this difference is only significant between CPP and control group, and there is no significant pairwise difference between IPA group or IPT group with other classified groups (Table 1). Also vitamin D levels are compared and no significant difference is observed between classified groups (Table 1).

Table 1

Demographic characteristics and hormonal laboratory values between groups

\begin{tabular}{|c|c|c|c|c|c|c|}
\hline Variables & $\begin{array}{l}\text { Controls(n } \\
=62 \text { ) } \\
\text { (mean } \pm \\
\text { SD) }\end{array}$ & $\begin{array}{l}\text { **CPP }(\mathrm{n}= \\
93) \\
(\mathrm{mean} \pm \\
\text { SD) }\end{array}$ & $\begin{array}{l}\text { IPT }(n=16) \\
(\text { mean } \pm \\
\text { SD) }\end{array}$ & $\begin{array}{l}\text { IPA(n= 12) } \\
(\text { mean } \pm \\
\text { SD) }\end{array}$ & $\begin{array}{l}\text { Total }(\mathrm{n}= \\
\text { 183) } \\
\text { (mean } \pm \\
\text { SD) }\end{array}$ & $\begin{array}{l}\mathrm{P} \\
\text { value }\end{array}$ \\
\hline $\begin{array}{l}\text { Chronological } \\
\text { age }(\mathrm{m})\end{array}$ & $\begin{array}{l}86.69 \pm \\
9.79\end{array}$ & $\begin{array}{l}88.96 \pm \\
13.40\end{array}$ & $\begin{array}{l}88.50 \pm \\
18.75\end{array}$ & $\begin{array}{l}85.00 \pm \\
12.350\end{array}$ & $\begin{array}{l}87.89 \pm \\
12.75\end{array}$ & 0.605 \\
\hline Bone age $(\mathrm{m})$ & $\begin{array}{l}85.51 \pm \\
14.40^{\mathrm{a}^{\star}}\end{array}$ & $\begin{array}{l}107.76 \pm \\
19.81^{b}\end{array}$ & $\begin{array}{l}91.43 \pm \\
20.92^{\mathrm{a}}\end{array}$ & $\begin{array}{l}94.16 \pm \\
9.04^{\mathrm{a}}\end{array}$ & $\begin{array}{l}97.90 \pm \\
20.38\end{array}$ & $<.001$ \\
\hline Height (cm) & $\begin{array}{l}124.15 \pm \\
8.70^{\star a}\end{array}$ & $\begin{array}{l}129.53 \pm \\
8.97^{b}\end{array}$ & $\begin{array}{l}126.53 \pm \\
11.58^{\mathrm{a}}\end{array}$ & $\begin{array}{l}123.75 \pm \\
8.59^{\mathrm{a}}\end{array}$ & $\begin{array}{l}127.07 \pm \\
9.43^{\mathrm{a}}\end{array}$ & 0.003 \\
\hline BMI & $\begin{array}{l}18.66 \pm \\
3.80\end{array}$ & $\begin{array}{l}19.44 \pm \\
3.74\end{array}$ & $\begin{array}{l}20.25 \pm \\
4.09\end{array}$ & $\begin{array}{l}18.69 \pm \\
3.94\end{array}$ & $\begin{array}{l}19.20 \pm \\
3.80\end{array}$ & 0.385 \\
\hline $\begin{array}{l}\text { Baseline LH } \\
(\mathrm{mlU} / \mathrm{ml})\end{array}$ & $0.26 \pm 0.34^{\mathrm{a}}$ & $1.35 \pm 2.13^{b}$ & $\begin{array}{l}0.42 \pm \\
0.43^{a b}\end{array}$ & $\begin{array}{l}0.22 \pm \\
0.07^{a b}\end{array}$ & $0.82 \pm 1.63$ & $<.001$ \\
\hline $\begin{array}{l}\text { Baseline FSH } \\
(\mathrm{mlU} / \mathrm{ml})\end{array}$ & $2.36 \pm 1.58^{\mathrm{a}}$ & $3.43 \pm 2.60^{\mathrm{b}}$ & $\begin{array}{l}2.85 \pm 2.43 \\
a b\end{array}$ & $\begin{array}{l}2.30 \pm 1.27 \\
a b\end{array}$ & $2.94 \pm 2.26$ & 0.024 \\
\hline Estradiol (pg/ml) & $8.65 \pm 7.58^{\mathrm{a}}$ & $\begin{array}{l}22.33 \pm \\
7.58^{\mathrm{b}}\end{array}$ & $\begin{array}{l}10.63 \pm \\
6.74 \mathrm{ab}\end{array}$ & $\begin{array}{l}5.23 \pm \\
1.74^{\mathrm{a}}\end{array}$ & $\begin{array}{l}15.55 \pm \\
20.94\end{array}$ & $\begin{array}{l}<.001 \\
0.001\end{array}$ \\
\hline $\begin{array}{l}\text { Vitamin D } \\
\text { (ng/mL) }\end{array}$ & $\begin{array}{l}27.68 \pm \\
16.72\end{array}$ & $\begin{array}{l}27.86 \pm \\
20.15\end{array}$ & $\begin{array}{l}34.12 \pm \\
16.60\end{array}$ & $\begin{array}{l}24.65 \pm \\
11.56\end{array}$ & $\begin{array}{l}28.16 \pm \\
18.40\end{array}$ & 0.664 \\
\hline \multicolumn{7}{|c|}{$\begin{array}{l}\text { *One-Way ANOVA was used for data analyzing; Dissimilar values }(a, b) \text { in each rows are significantly } \\
\text { different based on the Tukey's Test in post-hoc analysis. }\end{array}$} \\
\hline $\begin{array}{l}\text { **CPP: central pre } \\
\text { adrenarche, Basel } \\
\text { hormone BMI: boc }\end{array}$ & $\begin{array}{l}\text { ious puberty } \\
\text { LH: baseline } \\
\text { nass index. }\end{array}$ & $\begin{array}{l}\text { T: idiopathi } \\
\text { teinizing hor }\end{array}$ & $\begin{array}{l}\text { emature th } \\
\text { ne baselin }\end{array}$ & $\begin{array}{l}\text { che; IPA: idi } \\
\text { H: baseline }\end{array}$ & $\begin{array}{l}\text { athic prema } \\
\text { licle-stimul }\end{array}$ & \\
\hline
\end{tabular}


- Comparison of pelvic ultrasonography parameters

According to our one-way analysis of variance and post hoc analysis, CPP patients had significantly ( $\mathrm{p}<$ 0.001) increased uterine fundus AP diameter, uterine body AP diameter, F/C ratio, uterine length, uterine volume, endometrial thickness, transverse uterine diameter compared to other classified groups. Three parameters including uterine cervix AP diameter, left ovarian volume and right ovarian volume, only showed significantly higher values in CPP patients compared to control and IPA groups, whereas no significant differences are found between CPP patients and IPT patients in recent noted three parameters despite having higher values in CPP patients compared to IPT patients (Table 2). The average maximal diameter of the largest follicle only had a significant difference between CPP patients $(5.00 \pm 6.08 \mathrm{~mm})$ compared to the control group $(2.60 \pm 2.46 \mathrm{~mm})$ and when comparing this parameter in IPA and IPT groups with each other or with control or CPP groups, no significant difference was evident (Table 2). All sonographic parameters had no significant differences in control, IPA, IPT groups when compared to each other according to pairwise post-hoc analysis (Table 2). 
Table 2

Pelvic ultrasonography parameters between groups

\begin{tabular}{|c|c|c|c|c|c|c|}
\hline Variables & $\begin{array}{l}\text { Controls(n } \\
=62 \text { ) } \\
\text { (mean } \pm \\
\text { SD) }\end{array}$ & $\begin{array}{l}\text { **CPP }(\mathrm{n} \\
=93) \\
\text { (mean } \pm \\
\text { SD) }\end{array}$ & $\begin{array}{l}\text { IPT }(n= \\
16) \\
(m e a n \pm \\
\text { SD) }\end{array}$ & $\begin{array}{l}\text { IPA( } n= \\
12) \\
\text { (mean } \pm \\
\text { SD) }\end{array}$ & $\begin{array}{l}\text { Total }(n= \\
\text { 183) } \\
\text { (mean } \pm \\
\text { SD) }\end{array}$ & $\begin{array}{l}\mathrm{P} \\
\text { value }\end{array}$ \\
\hline $\begin{array}{l}\text { Uterine fundus AP } \\
\text { diameter(mm) }\end{array}$ & $\begin{array}{l}5.33 \pm \\
1.92^{\mathrm{a}}\end{array}$ & $\begin{array}{l}8.60 \pm \\
3.95^{b}\end{array}$ & $\begin{array}{l}5.00 \pm \\
1.44^{\mathrm{a}}\end{array}$ & $\begin{array}{l}4.55 \pm \\
1.25^{\mathrm{a}}\end{array}$ & $\begin{array}{l}6.91 \pm \\
3.52\end{array}$ & $<.001$ \\
\hline $\begin{array}{l}\text { Uterine body AP } \\
\text { diameter(mm) }\end{array}$ & $\begin{array}{l}5.90 \pm 2.01 \\
a\end{array}$ & $\begin{array}{l}8.26 \pm \\
3.49^{b}\end{array}$ & $\begin{array}{l}5.82 \pm \\
1.39^{a}\end{array}$ & $\begin{array}{l}4.85 \pm \\
1.40^{a}\end{array}$ & $\begin{array}{l}7.02 \pm \\
3.07\end{array}$ & $<.001$ \\
\hline $\begin{array}{l}\text { Uterine cervix AP } \\
\text { diameter(mm) }\end{array}$ & $\begin{array}{l}6.00 \pm \\
1.51^{\mathrm{a}}\end{array}$ & $\begin{array}{l}7.34 \pm \\
2.51^{\mathrm{b}}\end{array}$ & $\begin{array}{l}6.21 \pm \\
0.836^{\mathrm{ab}}\end{array}$ & $\begin{array}{l}5.54 \pm \\
1.01^{\mathrm{a}}\end{array}$ & $\begin{array}{l}6.67 \pm \\
2.14\end{array}$ & $<.001$ \\
\hline $\mathrm{F} / \mathrm{C}$ ratio & $\begin{array}{l}0.90 \pm 0.28 \\
\mathrm{a}\end{array}$ & $\begin{array}{l}1.16 \pm \\
0.323^{b}\end{array}$ & $\begin{array}{l}0.79 \pm \\
0.17^{a}\end{array}$ & $\begin{array}{l}0.84 \pm \\
0.32^{a}\end{array}$ & $\begin{array}{l}1.02 \pm \\
0.33\end{array}$ & $<.001$ \\
\hline Uterine length & $\begin{array}{l}28.96 \pm \\
4.81^{\mathrm{a}}\end{array}$ & $\begin{array}{l}38.04 \pm \\
8.33^{b}\end{array}$ & $\begin{array}{l}31.03 \pm \\
5.27^{\mathrm{a}}\end{array}$ & $\begin{array}{l}32.16 \pm \\
3.51^{\mathrm{a}}\end{array}$ & $\begin{array}{l}33.96 \pm \\
7.48\end{array}$ & $<.001$ \\
\hline Uterine volume & $\begin{array}{l}1.13 \pm 0.95 \\
\mathrm{a}\end{array}$ & $\begin{array}{l}3.13 \pm \\
2.64^{\mathrm{b}}\end{array}$ & $\begin{array}{l}1.16 \pm \\
0.38^{a}\end{array}$ & $\begin{array}{l}0.96 \pm \\
0.51^{\mathrm{a}}\end{array}$ & $\begin{array}{l}2.34 \pm \\
3.03\end{array}$ & $<.001$ \\
\hline Endometrial thickness & $\begin{array}{l}0.37 \pm 0.30 \\
\text { a }\end{array}$ & $\begin{array}{l}1.09 \pm \\
0.69^{b}\end{array}$ & $\begin{array}{l}0.45 \pm \\
0.16^{a}\end{array}$ & $\begin{array}{l}0.35 \pm \\
0.13^{a}\end{array}$ & $\begin{array}{l}0.80 \pm \\
0.83\end{array}$ & $<.001$ \\
\hline L-Ovarian volume (mm) & $\begin{array}{l}1.08 \pm 0.64 \\
a\end{array}$ & $\begin{array}{l}1.94 \pm \\
1.47^{b}\end{array}$ & $\begin{array}{l}1.45 \pm \\
0.92 \mathrm{ab}\end{array}$ & $\begin{array}{l}1.08 \pm \\
0.77^{a}\end{array}$ & $\begin{array}{l}1.55 \pm \\
1.23\end{array}$ & $<0.001$ \\
\hline R-Ovarian volume (mm) & $\begin{array}{l}1.10 \pm 0.73 \\
\mathrm{a}\end{array}$ & $\begin{array}{l}2.01 \pm \\
1.14^{\mathrm{b}}\end{array}$ & $\begin{array}{l}1.51 \pm \\
0.83 \mathrm{ab}\end{array}$ & $\begin{array}{l}1.14 \pm \\
0.74^{\mathrm{a}}\end{array}$ & $\begin{array}{l}1.60 \pm \\
1.05\end{array}$ & $<.001$ \\
\hline $\begin{array}{l}\text { Transverse uterine } \\
\text { diameter }(\mathrm{mm})\end{array}$ & $\begin{array}{l}11.66 \pm \\
4.12^{\mathrm{a}}\end{array}$ & $\begin{array}{l}17.02 \pm \\
6.49^{b}\end{array}$ & $\begin{array}{l}11.90 \pm \\
3.17^{\mathrm{a}}\end{array}$ & $\begin{array}{l}10.87 \pm \\
3.51^{\mathrm{a}}\end{array}$ & $\begin{array}{l}14.35 \pm \\
6.00\end{array}$ & $<.001$ \\
\hline $\begin{array}{l}\text { AMD of largest } \\
\text { follicle }(\mathrm{mm})\end{array}$ & $\begin{array}{l}2.60 \pm 2.46 \\
\mathrm{a}\end{array}$ & $\begin{array}{l}5.00 \pm \\
6.08^{b}\end{array}$ & $\begin{array}{l}3.90 \pm \\
2.19 \mathrm{ab}\end{array}$ & $\begin{array}{l}4.25 \pm \\
1.81 \mathrm{ab}\end{array}$ & $\begin{array}{l}4.04 \pm \\
4.74\end{array}$ & 0.022 \\
\hline \multicolumn{7}{|c|}{$\begin{array}{l}\text { *One-Way ANOVA was used for data analyzing; Dissimilar values }(a, b) \text { in each rows are significantly } \\
\text { different based on the Tukey's Test in post-hoc analysis. }\end{array}$} \\
\hline
\end{tabular}

- ROC analysis and cut off value determination for sonographic parameters

ROC analysis was done with each pelvic sonography parameter as an independent measurement between CPP patients and other groups (Fig. 1).Among all parameters three of them showed acceptable 
and excellent sensitivity and or specificity for identifying CPP patients from other groups. Uterine volume was one of these parameters which identified CPP patients from other groups by a cut off of $1.40 \mathrm{ml}$ (sensitivity $=75.27 \%$, specificity $=75.56 \%$, AUC $=0.826$ and SE $=0.030$ ). The other two parameters which had acceptable sensitivity and specificity were transverse uterine diameter (cut off of $13.5 \mathrm{~mm}$, sensitivity $=72.04 \%$, specificity $=71.11 \%, \mathrm{AUC}=0.780$ and $\mathrm{SE}=0.034$ ) F/C ratio (cut off of 0.98 , sensitivity $=78.49 \%$, specificity $=70 \%, A U C=0.788$ and $\mathrm{SE}=0.034)($ Table 3$)$. ROC analysis was also done between CPP patients and only control group (Fig. 2). In this analysis same three parameters were found to be most practical among the others. First of them was uterine volume with cut off of $1.40 \mathrm{ml}$ (sensitivity $=75.27 \%$, specificity $=74.19 \%, A U C=0.826$ and $S E=0.032$ ). The other two parameters were transverse uterine diameter (cut off of $13.5 \mathrm{~mm}$, sensitivity $=72.04 \%$, specificity $=72.58 \%, \mathrm{AUC}=0.778$ and SE $=0.037$ ) F/C ratio (cut off of 1 , sensitivity $=77.42 \%$, specificity $=69.35 \%$, AUC $=0.769$ and SE $=0.039)($ Table 4 ).

Table 3

Receiver-operating characteristics (ROC) curve parameters of study variables for identifying patients with central precocious puberty (CPP) from other groups.

\begin{tabular}{|c|c|c|c|c|c|c|c|}
\hline Parameter & $\begin{array}{l}\text { Area under } \\
\text { ROC curve }\end{array}$ & SE & $\begin{array}{l}\text { Cut-off } \\
\text { point }\end{array}$ & $\begin{array}{l}\text { Sensitivity } \\
\text { (\%) }\end{array}$ & $\begin{array}{l}\text { Specificity } \\
(\%)\end{array}$ & *LR+ & *LR- \\
\hline $\begin{array}{l}\text { Uterine fundus } \\
\text { AP diameter }\end{array}$ & 0.802 & 0.032 & 5.50 & 80.65 & 62.22 & 2.134 & 0.311 \\
\hline $\begin{array}{l}\text { Uterine body AP } \\
\text { diameter }\end{array}$ & 0.733 & 0.037 & 6.30 & 66.67 & 64.44 & 1.875 & 0.517 \\
\hline $\begin{array}{l}\text { Uterine cervix AP } \\
\text { diameter }\end{array}$ & 0.678 & 0.039 & 6.20 & 63.44 & 66.67 & 1.903 & 0.548 \\
\hline $\mathrm{F} / \mathrm{C}$ ratio & 0.788 & 0.034 & 0.98 & 78.49 & 70.00 & 2.616 & 0.307 \\
\hline Uterine length & 0.842 & 0.028 & 35.00 & 70.97 & 82.22 & 3.991 & 0.353 \\
\hline Uterine volume & 0.826 & 0.030 & 1.40 & 75.27 & 75.56 & 3.079 & 0.327 \\
\hline $\begin{array}{l}\text { Endometrial } \\
\text { thickness }\end{array}$ & 0.675 & 0.026 & 1.00 & 37.63 & 96.67 & 11.290 & 0.645 \\
\hline L-Ovarian volume & 0.722 & 0.037 & 1.30 & 66.30 & 63.33 & 1.808 & 0.532 \\
\hline R-Ovarian volume & 0.748 & 0.036 & 1.10 & 78.49 & 61.11 & 2.018 & 0.351 \\
\hline $\begin{array}{l}\text { Transverse } \\
\text { uterine diameter }\end{array}$ & 0.780 & 0.034 & 13.5 & 72.04 & 71.11 & 2.493 & 0.393 \\
\hline $\begin{array}{l}\text { AMD of largest } \\
\text { follicle }\end{array}$ & 0.611 & 0.041 & 4.00 & 61.29 & 52.22 & 1.282 & 0.741 \\
\hline
\end{tabular}


Table 4

Receiver-operating characteristics (ROC) curve parameters of study variables for identifying patients with central precocious puberty (CPP) from control group.

\begin{tabular}{|c|c|c|c|c|c|c|c|}
\hline Parameter & $\begin{array}{l}\text { Area under } \\
\text { ROC curve }\end{array}$ & SE & $\begin{array}{l}\text { Cut-off } \\
\text { point }\end{array}$ & $\begin{array}{l}\text { Sensitivity } \\
\text { (\%) }\end{array}$ & $\begin{array}{l}\text { Specificity } \\
(\%)\end{array}$ & *LR+ & *LR- \\
\hline $\begin{array}{l}\text { Uterine fundus AP } \\
\text { diameter }\end{array}$ & 0.785 & 0.036 & 6.50 & 68.82 & 75.81 & 2.844 & 0.411 \\
\hline $\begin{array}{l}\text { Uterine body AP } \\
\text { diameter }\end{array}$ & 0.718 & 0.040 & 6.30 & 66.67 & 61.29 & 1.722 & 0.543 \\
\hline $\begin{array}{l}\text { Uterine cervix AP } \\
\text { diameter }\end{array}$ & 0.676 & 0.043 & 6.50 & 61.29 & 70.97 & 2.111 & 0.545 \\
\hline $\mathrm{F} / \mathrm{C}$ ratio & 0.769 & 0.039 & 1.00 & 77.42 & 69.35 & 2.526 & 0.325 \\
\hline Uterine length & 0.871 & 0.027 & 33.00 & 79.57 & 72.58 & 2.902 & 0.281 \\
\hline Uterine volume & 0.826 & 0.032 & 1.40 & 75.27 & 74.19 & 2.916 & 0.333 \\
\hline $\begin{array}{l}\text { Endometrial } \\
\text { thickness }\end{array}$ & 0.893 & 0.028 & 1.00 & 37.63 & 95.16 & 7.777 & 0.655 \\
\hline L-Ovarian volume & 0.744 & 0.039 & 1.2 & 69.57 & 61.29 & 1.797 & 0.496 \\
\hline R-Ovarian volume & 0.776 & 0.038 & 1.2 & 76.34 & 69.35 & 2.491 & 0.341 \\
\hline $\begin{array}{l}\text { Transverse uterine } \\
\text { diameter }\end{array}$ & 0.778 & 0.037 & 13.5 & 72.04 & 72.58 & 2.627 & 0.385 \\
\hline $\begin{array}{l}\text { AMD of largest } \\
\text { follicle }\end{array}$ & 0.650 & 0.042 & 4.00 & 61.29 & 61.29 & 1.583 & 0.631 \\
\hline
\end{tabular}

- Checking the equality of the ROC area of each parameter against a "gold standard" ROC curve

According to our study and most of other studies, uterine volume had a significant and constant value as a diagnostic parameter for recognizing CPP patients. Among all parameters, the best parameter was uterine volume, as selected by the highest value of area under the ROC curve and had acceptable sensitivity and specificity. That is why we check the equality of the ROC area of other sonography parameters compared to the ROC area of uterine volume. As a result area under the ROC curve of all parameters except uterine body AP diameter, uterine cervix AP diameter, and average maximal diameter of the largest follicle of both ovaries had no significant difference with the gold standard curve, which means all sonography parameters except noted parameters above had equal diagnostic value compared to the gold standard at both study designs (CPP toward others and CPP toward only control group) (Table 5). 
Table 5

ROC area of each parameter against uterine volume ROC curve as gold standard ROC curve

\begin{tabular}{|lllllll|}
\hline Parameter & $\begin{array}{l}\text { Area under ROC } \\
\text { curve }\end{array}$ & SE & Chi2 & df & $\begin{array}{c}\text { PR }> \\
\text { chi2 }\end{array}$ & $\begin{array}{l}\text { Bonferroni PR }> \\
\text { chi2 }\end{array}$ \\
\hline $\begin{array}{l}\text { Uterine volume } \\
\text { (standard) }\end{array}$ & 0.826 & 0.030 & & & & \\
\hline $\begin{array}{l}\text { Uterine fundus AP } \\
\text { diameter }\end{array}$ & 0.802 & 0.032 & 0.901 & 1 & 0.342 & 1 \\
\hline $\begin{array}{l}\text { Uterine body AP } \\
\text { diameter }\end{array}$ & 0.733 & 0.037 & 13.455 & 1 & 0.000 & 0.002 \\
\hline $\begin{array}{l}\text { Uterine cervix AP } \\
\text { diameter }\end{array}$ & 0.678 & 0.039 & 21.707 & 1 & 0.000 & 0.000 \\
\hline F/C ratio & 0.788 & 0.034 & 1.180 & 1 & 0.277 & 1 \\
\hline Uterine length & 0.842 & 0.028 & 0.232 & 1 & 0.629 & 1 \\
\hline Endometrial thickness & 0.892 & 0.024 & 4.049 & 1 & 0.044 & 0.441 \\
\hline L-Ovarian volume & 0.722 & 0.037 & 7.468 & 1 & 0.006 & 0.062 \\
\hline R-Ovarian volume & 0.748 & 0.036 & 4.677 & 1 & 0.030 & 0.305 \\
\hline $\begin{array}{l}\text { Transverse uterine } \\
\text { diameter }\end{array}$ & 0.780 & 0.034 & 4.630 & 1 & 0.031 & 0.314 \\
\hline AMD of largest follicle & 0.611 & 0.041 & 18.994 & 1 & 0.000 & 0.000 \\
\hline
\end{tabular}

\section{Discussion}

The gold standard of CPP diagnosis is the GnRH-stimulation test, which has different cut-off values according to different investigations (19-21). Moreover, this test is a relatively high cost and timeconsuming test, also despite its high specificity, it has a low sensitivity (16-23). Meanwhile, pelvic sonography is a useful, non-invasive, and relatively low-cost method for assessing female pelvic parameters (uterus and ovaries)(13). In this study we used clinical and laboratory data for the classification of cases. All cases were classified in either one of the control, IPA, IPT, or CPP groups. The criteria proposed by Kim et al. (19), according to which a stimulated LH level of $\geq 5 \mathrm{IU} / \mathrm{L}$ is considered as the gold standard to the diagnosis of CPP patients. In addition, at least two years of follow-up of the patients is done to lower the possibility of misdiagnosis of patients' classification (especially IPA, IPT forms of precocious puberty $)(9,21)$. We analyzed the difference in pelvic sonography parameters between CPP, IPA, IPT, and control groups and calculated cutoff values to differentiate CPP patients from other groups. To our knowledge, few studies compare all these classified groups with each other $(14,22,24)$. It is also original in this study that cut off values were extracted from this study to differentiate $\mathrm{GnRH}$ treatment needed group (CPP group) from no treatment needed group (other three groups), and also for the first time we checked the equality of the ROC area of each parameter against a gold standard ROC 
curve (uterine volume) as judged by the biggest value of area under the ROC curve with acceptable sensitivity and specificity. Our study found that most of the pelvic sonography parameters were useful to distinguish CPP from other groups. Among all of the parameters, uterine volume as the best diagnostic parameter and three other parameters including; uterine transverse diameter, F/C ratio, and endometrial thickness showed an important role in the optimal diagnosis of CPP patients (Table 3 and Table 4). The cutoff values for uterine volume, which identified CPP patients from other groups and only from the control group was $1.40 \mathrm{ml}$ with sensitivities of $75.27 \%$ and $75.27 \%$ and specificities of $75.56 \%$ and $74.19 \%$, respectively. Most of the previous studies like ours declared uterine volume as the best parameter for CPP diagnosis but there were differences in uterine volume cut-off values. Wen et al. and $\mathrm{Yu} \mathrm{J}$ et al. respectively reported $91.66 \%$ and $59.1 \%$ sensitivities and $77.60 \%$ and $71.0 \%$ specificities for the cutoff value of $1.09 \mathrm{~mL}$ and $1.07 \mathrm{ml}$ of uterine volume $(22,25)$. On the other hand higher values with acceptable sensitivity and specificity were reported by Haber et al. (cut off value of $1.8 \mathrm{~mL}$ with a $100 \%$ sensitivity and specificity for the uterine volume) (16). De Vries et al. (cut off value of $1.96 \mathrm{ml}$ with a sensitivity of $88.8 \%$ and specificity of $89.4 \%$ )(26), and Battaglia et al (cut off value of $4 \mathrm{ml}$ with a sensitivity of $87 \%$ and specificity of $87.5 \%)(27)$. These results show the most powerful sonography parameter and its 'optimal cutoff value may differ according to ethnicity differences, sample size, and type of different studies. The second and third most efficient parameters in our study were uterine transverse diameter and F/C ratio with a cut off of $13.5 \mathrm{~mm}(72.04 \%$ sensitivity and $71.11 \%$ specificity) and $0.98(78.49 \%$ sensitivity and $70.00 \%$ specificity) respectively, for CPP against other groups and with a cut-off of $13.5 \mathrm{~mm}$ ( $72.04 \%$ sensitivity and $72.58 \%$ specificity) and 1 ( $77.42 \%$ sensitivity and $69.35 \%$ specificity) respectively, for CPP against the only control group. These findings are in close similarity with $\mathrm{Yu} J$ et al. (25) and De Vries et al. (26) findings in transverse uterine diameter and in close similarity with Badouraki et al. (24), De Vries et al. (26), and Binay et al.(28), findings in F/C ratio parameter. Our study also suggests that endometrial thickness and echogenicity had a high specificity in diagnosing CPP patients, which is reported as the same in most of the studies $(26,27,29)$ and in comparison with Wen et al. findings (22). Previous studies in different countries found ovarian volume having a good diagnostic value for identifying the CPP patients $(15,24,26,30)$. In our study despite the significant difference between CPP patients compared to control and IPA groups, there was no significant difference between CPP patients compared to the IPT group. That is why we don't suggest ovarian volume as a discriminate parameter to differentiate CPP from other groups. This suggestion is in agreement with some other studies $(16,22,27,31)$. At the end, we compared all sonography parameters to the uterine volume ROC curve, as selected by the highest value of area under the ROC curve and had acceptable sensitivity and specificity. This analysis helped us to classified pelvic sonography parameters as in equal diagnostic value to uterine volume (such as uterine fundus AP diameter, F/C ratio, uterine length, uterine volume, endometrial thickness, transverse uterine diameter, and ovarian volume) and not equal diagnostic value to uterine volume (such as uterine body AP diameter, uterine cervix AP diameter and average maximal diameter of the largest follicle of both ovaries) (Table 5).

\section{Conclusion}


On-time beginning of GnRH agonist treatment in CPP patients is crucial for preventing early bone maturation, reduced final height, and related physiological stress effects. Also, differentiate CPP patients from IPA and IPT groups is important in the clinical field to avoid unnecessary treatment for CPP-like groups (IPA and IPT). In this study, we evaluate female sonography parameters in correlation with precise clinical and laboratory data. Comparison of all sonography parameters in different groups showed that uterine volume, uterine transverse diameter, $\mathrm{F} / \mathrm{C}$ ratio, and endometrial thickness are good predictors of central precocious puberty. On the other hand, we found that ovarian volume is only helpful in differentiate CPP patients from normal control cases, and using this parameter as a distinguishing factor between treatment needed (CPP patients) and no treatment needed (IPA, IPT, control groups) classification is not accurate.

Our study is limited due to the retrospective and cross-sectional nature of the study design, in which we do not have control over confounding factors such as ethnicity differences and sample drop in case files. Also due to the small number of under six year's old patients, we don't evaluate cases in age ranged subgroups.

\section{Abbreviations}

CPP: Central precocious puberty

IPT: Isolated premature thelarche

IPA: Isolated premature adrenarche

GDPP: Gonadotropin dependent precocious puberty

GIPP: Gonadotropin independent precocious puberty

GnRH. Gonadotropin releasing hormone

E2: Estradiol

LH: Luteinizing hormone

FSH: Follicle stimulating hormone

US: Ultrasonography

HPG: Hypothalamic-pituitary-gonadal

AUC: Area under the curve

ROC: Receiver operating characteristic 


\section{Declarations}

\section{Acknowledgement}

The Authors would like to thank Ali Asghar Clinical Research Development Center (AACRDC), Iran University of Medical Sciences (IUMS) for their support to perform this project.

\section{Ethics approval and consent to participate}

All participants were informed about the study and confidentiality protocols. Written Informed consent was obtained from all the participants. The Ethics committee of Iran University of Medical Sciences confirmed all procedures of this study. (IR.IUMS.FMD.REC.1399.246)

\section{Consent for publication}

Not applicable.

\section{Availability of data and materials}

The datasets used and analyzed during the current study are available from the corresponding author on reasonable request.

\section{Competing interests}

The authors declare that they have no competing interests.

\section{Funding}

This study did not receive any funding for this work.

\section{Author Contribution}

EZ and MV (study conception \& design and Critical revision), NR and MK (Acquisition of data, Analysis and interpretation of data \& Drafting of manuscript), NR, AA and KM (Critical revision \& Analysis and interpretation of data)

\section{References}

1. Bridges NA, Christopher JA, Hindmarsh PC, Brook CG. Sexual precocity: sex incidence and aetiology. Arch Dis Child. 1994;70(2):116-8.

2. Klein KO. Precocious puberty: who has it? Who should be treated? J Clin Endocrinol Metab. 1999;84(2):411-4.

3. Tanner JM. Growth at adolescence, 2nd ed. Thomas: Springfield, III.; 1962. 
4. Parent AS, Teilmann G, Juul A, Skakkebaek NE, Toppari J, Bourguignon JP. The timing of normal puberty and the age limits of sexual precocity: variations around the world, secular trends, and changes after migration. Endocr Rev. 2003;24(5):668-93.

5. Grumbach MM. The neuroendocrinology of human puberty revisited. Horm Res. 2002;57 Suppl 2:214.

6. Brauner R, Adan L, Malandry F, Zantleifer D. Adult height in girls with idiopathic true precocious puberty. J Clin Endocrinol Metab. 1994;79(2):415-20.

7. Ehrhardt AA, Meyer-Bahlburg HF. Idiopathic precocious puberty in girls: long-term effects on adolescent behavior. Acta Endocrinol Suppl (Copenh). 1986;279:247-53.

8. Kauli R, Galatzer A, Kornreich L, Lazar L, Pertzelan A, Laron Z. Final height of girls with central precocious puberty, untreated versus treated with cyproterone acetate or $\mathrm{GnRH}$ analogue. A comparative study with re-evaluation of predictions by the Bayley-Pinneau method. Horm Res. 1997;47(2):54-61.

9. Salardi S, Cacciari E, Mainetti B, Mazzanti L, Pirazzoli P. Outcome of premature thelarche: relation to puberty and final height. Arch Dis Child. 1998;79(2):173-4.

10. Klein KO, Larmore KA, de Lancey E, Brown JM, Considine RV, Hassink SG. Effect of obesity on estradiol level, and its relationship to leptin, bone maturation, and bone mineral density in children. $J$ Clin Endocrinol Metab. 1998;83(10):3469-75.

11. Lee PA. Central precocious puberty. An overview of diagnosis, treatment, and outcome. Endocrinol Metab Clin North Am. 1999;28(4):901-18, xi.

12. lughetti L, Predieri B, Ferrari M, Gallo C, Livio L, Milioli S, et al. Diagnosis of central precocious puberty: endocrine assessment. J Pediatr Endocrinol Metab. 2000;13 Suppl 1:709-15.

13. Garel L, Dubois J, Grignon A, Filiatrault D, Van Vliet G. US of the pediatric female pelvis: a clinical perspective. Radiographics. 2001;21(6):1393-407.

14. Buzi F, Pilotta A, Dordoni D, Lombardi A, Zaglio S, Adlard P. Pelvic ultrasonography in normal girls and in girls with pubertal precocity. Acta Paediatr. 1998;87(11):1138-45.

15. Herter LD, Golendziner E, Flores JA, Moretto M, Di Domenico K, Becker E, Jr., et al. Ovarian and uterine findings in pelvic sonography: comparison between prepubertal girls, girls with isolated thelarche, and girls with central precocious puberty. J Ultrasound Med. 2002;21(11):1237-46; quiz 47-8.

16. Haber HP, Wollmann HA, Ranke MB. Pelvic ultrasonography: early differentiation between isolated premature thelarche and central precocious puberty. Eur J Pediatr. 1995;154(3):182-6.

17. Kelly A, Winer KK, Kalkwarf H, Oberfield SE, Lappe J, Gilsanz V, et al. Age-based reference ranges for annual height velocity in US children. J Clin Endocrinol Metab. 2014;99(6):2104-12.

18. Gaskin CM, Kahn SL, Bertozzi JC, Bunch PM. Skeletal Development of the Hand and Wrist: A Radiographic Atlas and Digital Bone Age Companion: Oxford University Press, USA; 2011.

19. Kim HK, Kee SJ, Seo JY, Yang EM, Chae HJ, Kim CJ. Gonadotropin-releasing hormone stimulation test for precocious puberty. Korean J Lab Med. 2011;31(4):244-9. 
20. Neely EK, Wilson DM, Lee PA, Stene M, Hintz RL. Spontaneous serum gonadotropin concentrations in the evaluation of precocious puberty. J Pediatr. 1995;127(1):47-52.

21. Palmert MR, Malin HV, Boepple PA. Unsustained or slowly progressive puberty in young girls: initial presentation and long-term follow-up of 20 untreated patients. J Clin Endocrinol Metab. 1999;84(2):415-23.

22. Wen $X$, Wen $D$, Zhang $H$, Zhang $H$, Yang Y. Observational study pelvic ultrasound a useful tool in the diagnosis and differentiation of precocious puberty in Chinese girls. Medicine (Baltimore). 2018;97(10):e0092.

23. Aritaki S, Takagi A, Someya H, Jun L. A comparison of patients with premature thelarche and idiopathic true precocious puberty in the initial stage of illness. Acta Paediatr Jpn. 1997;39(1):21-7.

24. Badouraki M, Christoforidis A, Economou I, Dimitriadis AS, Katzos G. Evaluation of pelvic ultrasonography in the diagnosis and differentiation of various forms of sexual precocity in girls. Ultrasound Obstet Gynecol. 2008;32(6):819-27.

25. Yu J, Shin HY, Lee SH, Kim YS, Kim JH. Usefulness of pelvic ultrasonography for the diagnosis of central precocious puberty in girls. Korean J Pediatr. 2015;58(8):294-300.

26. de Vries L, Horev G, Schwartz M, Phillip M. Ultrasonographic and clinical parameters for early differentiation between precocious puberty and premature thelarche. Eur $\mathrm{J}$ Endocrinol. 2006;154(6):891-8.

27. Battaglia C, Mancini F, Regnani G, Persico N, lughetti L, De Aloysio D. Pelvic ultrasound and color Doppler findings in different isosexual precocities. Ultrasound Obstet Gynecol. 2003;22(3):277-83.

28. Binay C, Simsek E, Bal C. The correlation between GnRH stimulation testing and obstetric ultrasonographic parameters in precocious puberty. J Pediatr Endocrinol Metab. 2014;27(1112):1193-9.

29. Eksioglu AS, Yilmaz S, Cetinkaya S, Cinar G, Yildiz YT, Aycan Z. Value of pelvic sonography in the diagnosis of various forms of precocious puberty in girls. J Clin Ultrasound. 2013;41(2):84-93.

30. Sathasivam A, Rosenberg HK, Shapiro S, Wang H, Rapaport R. Pelvic ultrasonography in the evaluation of central precocious puberty: comparison with leuprolide stimulation test. J Pediatr. 2011;159(3):490-5.

31. Griffin I, Cole T, Duncan K, Hollman A, Donaldson M. Pelvic ultrasound findings in different forms of sexual precocity. Acta Paediatrica. 1995;84(5):544-9.

\section{Figures}




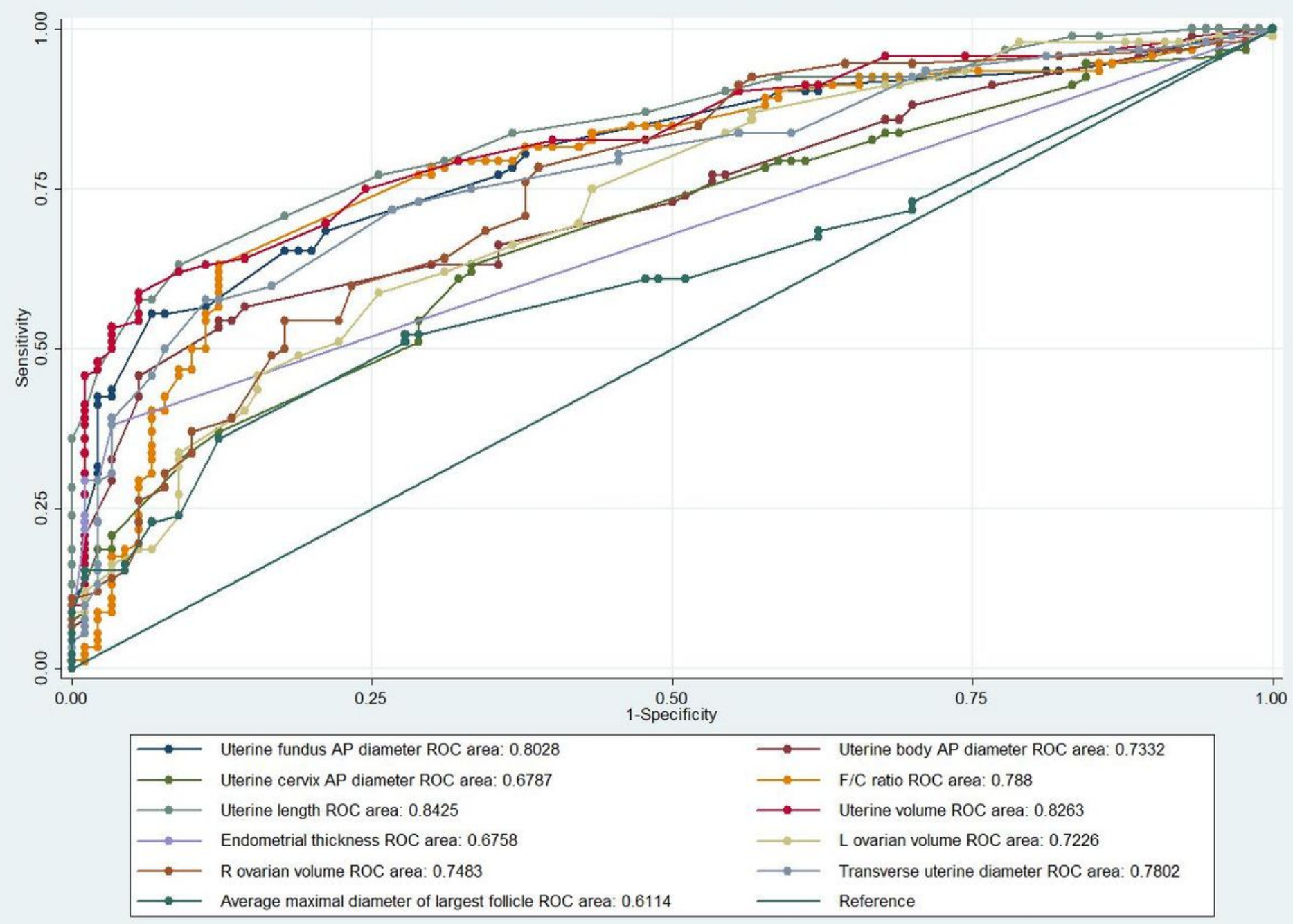

Figure 1

Receiver-operating characteristics (ROC) curve parameters of study variables for identifying patients with central precocious puberty (CPP) from other groups. 


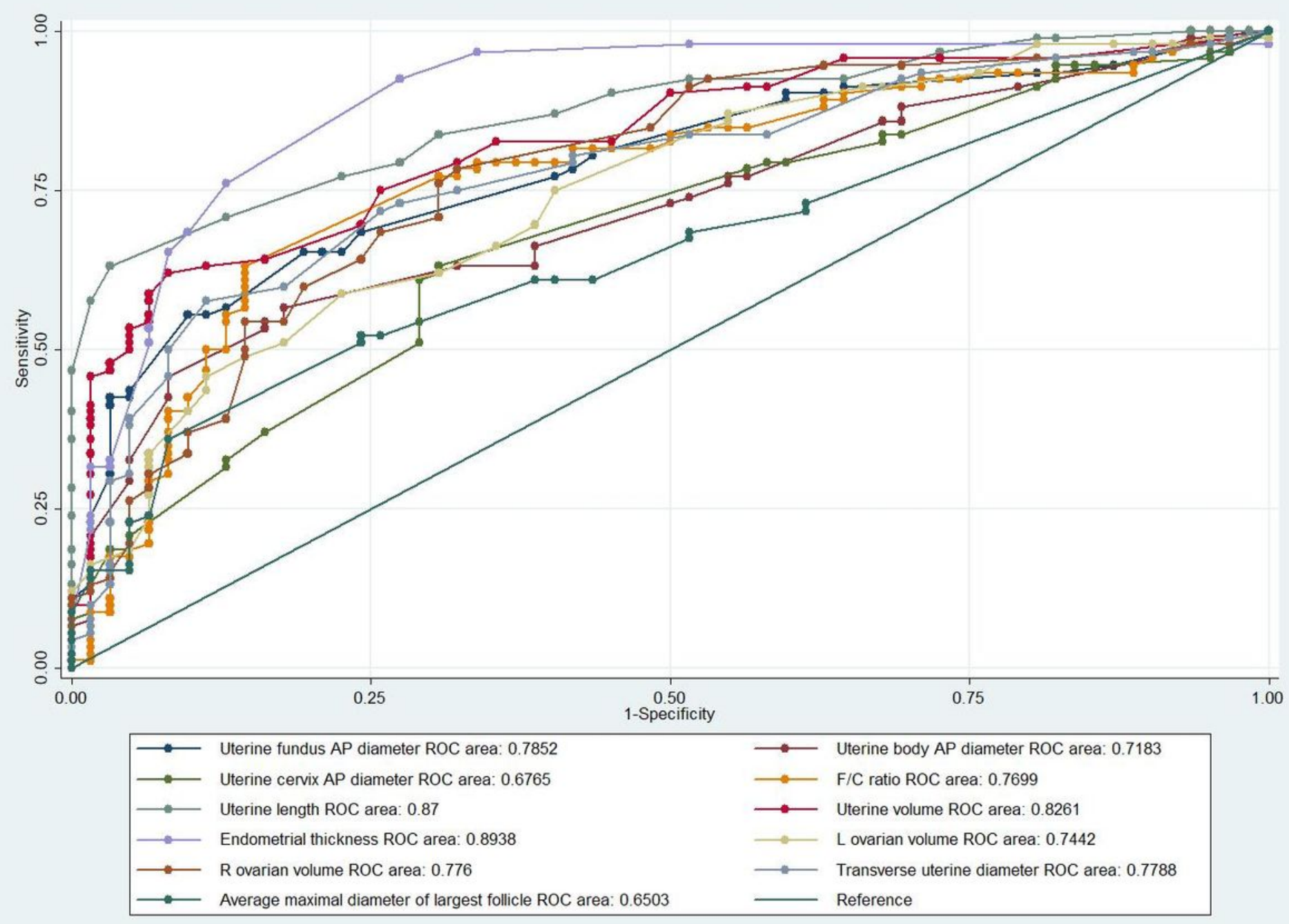

Figure 2

Receiver-operating characteristics (ROC) curve parameters of study variables for identifying patients with central precocious 